\title{
MONITORING ALPINE GLACIERS FROM CLOSE-RANGE TO SATELLITE SENSORS
}

\author{
V. Yordanov ${ }^{\text {a }}$, D. Fugazza ${ }^{\text {b }}$, R.S. Azzoni ${ }^{\text {b }}$, M. Cernuschi ${ }^{\text {c }}$, M. Scaioni ${ }^{\text {d, }}$, G.A Diolaiuti ${ }^{\text {b }}$
}

\author{
${ }^{a}$ Dept. of Civil and Environment Engineering (DICA), Politecnico di Milano \\ piazza L. da Vinci 32, 20133 Milano, Italy - email: vasil.yordanov@polimi.it \\ ${ }^{\mathrm{b}}$ Dept. of Environmental Science and Policy (DESP), Università degli studi di Milano \\ via Mangiagalli 34, 20133 Milano, Italy - email: \{davide.fugazza, robertosergio.azzoni, guglielmina.diolaiuti\}@unimi.it \\ c Agricola 2000 S.C.P.A., 20067 Tribiano (MI), Italy - email: massimo.cernuschi@gmail.com \\ ${ }^{\mathrm{d}}$ Dept. of Architecture, Built Environment and Construction Engineering (DABC), Politecnico di Milano \\ via Ponzio 31, 20133 Milano, Italy - email: marco.scaioni@polimi.it
}

\section{Commission III, WG III/9}

\begin{abstract}
:
In this paper the use of different types of remote-sensing techniques for monitoring topographic changes of Alpine glaciers is presented and discussed. Close range photogrammetry based on Structure-from-Motion approach is adopted to process images recorded from ground-based and drone-based stations in order to output dense point clouds. These are then directly compared to detect local changes by mean of M3C2 algorithm, while digital elevation models are interpolated to find global ice thinning and retreat. Medium-resolution satellite imagery can be exploited to monitor the glacier evolution at lower resolution but including the development and collapse of large crevasses. A case study concerning the Forni Glacier in the Raethian Alps (Italy) is presented to demonstrate the feasibility of the proposed approach by adopting data sets collected from 2016 to 2018.
\end{abstract}

KEY WORDS: Close-range Photogrammetry, Drone Photogrammetry, Forni Glacier, Glaciology, Medium-resolution, Near Sensing, Remote Sensing, Satellite Imagery, Sentinel 2, Structure-from-Motion, UAV/UAS.

\section{INTRODUCTION}

The impressive changes in the Alpine glaciers can be timely observed and studied on the basis of different types of imaging/scanning sensors that may be operated from ground-based, manned/unmanned airborne and spaceborne platforms. Section 2 will revise those remote sensing techniques that may be successfully applied to derive the 3D geometry of glacial and periglacial areas, even though other types of information may be exploited for further analysis (for example, the radiometric content of images or the laser intensity), as discussed in Fugazza (2019).

A case study concerning the Forni Glacier in the Raethian Alps (Italy) is presented here to show what may be obtained from different remote sensing data sets (Azzoni et al., 2017).

\section{REMOTE SENSING FOR ALPINE GLACIER MONITORING}

In the following some remote-sensing techniques that can be applied for monitoring the topographic changes of Alpine glaciers are presented and discussed. These have been classified according to the relative range between sensors and the glacier to be observed into close-/near-range sensing (Subsect. 2.1), airborne sensing (Subsect. 2.2), and spaceborne sensing (Subsect. 2.3).

\subsection{Close-/near-range sensing}

The term close-range has been used in the Photogrammetry since the 80's to address those projects where images are collected from ground-based stations, in general using large photo-scale if compared to Aerial Photogrammetry (see Atkinson, 1996; Luhmann et al., 2014). Thanks to development of terrestrial laser scanning (TLS - Vosselman and Maas, 2010) and the success of Structure-from-Motion (SfM - Granshaw, 2018b), in the latest 20 years this sector of photogrammetric 3D modelling (also called Terrestrial Photogrammetry) has significantly expanded. With the diffusion of drones (Giordan et al., 2018; Granshaw, 2018a), the possibility of reconstructing the terrain topography and the objects located on its surface by using high-resolution digital photos has impressively grown up. Mostly, the approach adopted to process the images acquired by drones is still based on SfM, which also allows to deal with less regular block geometry than in the case of standard aerial blocks (Barazzetti et al., 2010). For this reason, the term near sensing has been introduced to indicate those applications where images (or laser scans) are collected from ground or drones from a distance that is limited to a few hundred metres. Of course, the term near sensing also may be used when the information to collect is not applied for metric purpose, but to extract data for the classification of surface content, e.g., in the case of thermal and multi-/hyper-spectral sensors).

In the mountain environment, the application of close/near sensing may play an important role to study a wide range of processes where a very high-resolution is needed and when vertical or sub-vertical surfaces have to be reconstructed (see Rutzinger et al., 2016; 2018). In Scaioni et al. (2018) the use of SfM to reconstruct Alpine glaciers is addressed and some technical considerations reported. As discussed in Fugazza et al. (2018), the integration of images collected from ground-based station and images acquired from a drone is demonstrated to be a more complete way to describe the topography of an Alpine glacier.

After experiments carried out from 2016 to 2018 and comparing other experiences from the literature, the authors have come to define a complete, efficient and rapid approach 
that lead to georeferenced 3D models of Alpine glaciers (see Subsect. 3.1)

\subsection{Airborne remote Sensing}

Manned aircrafts still are the main platforms to operate photogrammetric projects for topographic mapping, including the use of LiDAR sensors (Shan and Toth, 2009). In general, specific missions for glacier monitoring are expensive to organize and difficult to be accomplished because of weather and orographic conditions. On the other hand, raw images to process or final products such as digital elevation models (DEMs) and orthoimages may be retrieved from regional projects. Though the image resolution is typically lower than in the case of close/near sensing campaigns, aerial photos may help compute the global volume of a glacier to be compared within different measurement epochs, if available (see Fugazza et al., 2018).

An interesting aspect related to aerial imagery is the chance to find archive photos that may be used to retrieve topographic information from the past decades (Walstra et al., 2007). Li et al. (2017) exploited some declassified satellite photographs to derive ice velocity and topography from the early 60's in Antarctica. Examples of processing such kind of data using SfM approach can be found in Cogliati et al. (2017) and Feurer and Vinatier (2018).

\subsection{Spaceborne remote sensing}

Despite of the fact that meteorological conditions may limit the number of images to process and the resolution is lower than the one typical of sensors adopted in close/near/airborne sensing, satellite imagery do not require the organization of specific missions and may cover large areas on the ground.

On one side, high-resolution imagery feature a ground sample distance (GSD) spanning from few tens of centimetres to some metres. The availability of stereo-pairs is exploitable to produce high-resolution digital surface models (DSMs). Unfortunately, this kind of data are still quite expensive, unless the case they are obtained under specific supporting or funding initiatives. Their cost, coupled with the difficulty of collecting cloud-free data sets, makes the analysis of high-resolution time-series over mountain glaciers a non-trivial task.

On the other, medium-resolution satellite imagery (e.g., Landsat, SPOT, Sentinel 2 constellations) feature GSDs spanning in the range between $10-30 \mathrm{~m}$ in optical bands, while they are delivered under open-data policy. The short repeat pass and the combination of more satellite/constellations allows to obtain long time-series consisting of more images per month. As it will be shown in Subsection 3.3, medium-resolution images offer the chance to evaluate the glacier extension (see also Paul et al., 2016), to make glacier inventories (see also Kääb et al., 2002), to identify the development of large collapsed areas, while they cannot used to detect elevation changes. In other studies it has been shown how these data sets can be exploited to derive ice-flow velocity also in the case of Alpine glaciers.

\section{APPLICATION TO FORNI GLACIER}

In recent years the Forni Glacier (Ortles Cevedale group, Rhaetian Alps) located in the National Stelvio Park (Italy) has been deeply investigated using remote-sensing techniques (see Fugazza et al., 2018; Scaioni et al., 2018; Fugazza, 2019). These analyses have continued a series of observations recorded during previous decades when a progressive thinning has happened (Diolaiuti and Smiraglia, 2010). In particular, the terminus of the glacier tongue has undergone impressive local disruption processes (Azzoni et al., 2017), which have been documented in remotely-sensed data. In Figure 1 an example of what happened at the end of Summer 2017 at the hydrographic right side of the glacier terminus is shown.

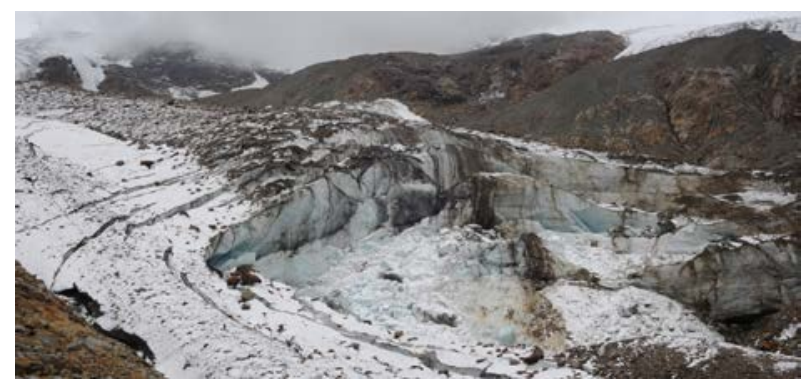

Figure 1. A large collapse occurred on the terminus of Forni Glacier in the end of Summer 2017.

In the following subsections digital data collected at Forni Glacier by using remote-sensing techniques are illustrated and compared. We limit here the analysis to geometric aspects, but other types of investigations have been carried out as well, e.g., ice-surface albedo (Azzoni et al., 2016; 2018; Fugazza et al., 2016). These data sets will be useful to better understand the local glacier dynamics and to investigate the precursory signals, of local collapses, which may result in severe consequences to the hikers and tourists which visit the glacier during summer time (Scaioni et al., 2019).

At the moment, no aerial photos have been used but data mining in existing archives is ongoing for future exploitation.

\subsection{SfM from terrestrial and drone platforms}

After a series of repeated yearly campaigns, a methodology for the near sensing of the Forni Glacier is now clear. During this study the comparison between different types of sensors for reconstruction of DSMs was also entailed. In the and, the use of SfM (Eltner et al., 2015) has been preferred, also because of its simpler use with respect to TLS (Fugazza et al., 2018). While more technical details are discussed in Scaioni et al. (2018), here we limit to draw a few guidelines of the adopted 3D image-based surveying pipeline. Details about mission planning, data acquisition parameter setup and adopted sensors can be found in the previous quoted papers. For a general view about these aspects the reader may also see Pepe et al. (2019), Mosbrucker et al. (2017), and O’Connor et al. (2017).

Two different data acquisition platforms were used with the aim of providing data to a common processing workflow based on SfM:

1. Terrestrial photogrammetry to capture the whole front of the glacier terminus, with the specific focus of reconstructing vertical and sub-vertical ice surfaces; 
2. Drone-based photogrammetry using a customized quadcopter, which flew over the ice tongue, a part of the periglacial area in front and at both flanks of the glacier.

Data sets obtained from both platforms at each measurement epoch were independently processed to obtain point clouds. Agisoft Photoscan ${ }^{\circledR}$ (and then Metashape ${ }^{\circledR}$ ) were adopted to apply SfM to both terrestrial and drone images.

In order to define the georeferencing of all point clouds in the same geodetic reference system and to allow the comparison between data sets, different solutions based on the use of ground control points (GCPs) were evaluated. The easier way to include GPCs in the photogrammetric project resulted to be the adoption of targets to be deployed on the ground/glacier surface. Targets' coordinates were measured at each campaign using a GNSS-RTK rover sensor and a master station located on a stable point in the proximity of the glacier. Such kind of targets (about 7-10 per campaign) could be clearly recognized in images from drone, but not in terrestrial photos. After trying solutions based on total station measurements of another set of GCPs for terrestrial blocks (see Scaioni et al., 2017) or trying to directly determine the position of camera stations using GNSS as proposed in Forlani et al. (2015), we abandoned these approaches because too much involved to handle in the glacier environment. Thus, the georeferencing of each "terrestrial" point cloud has been done by the co-registration to the contemporary point cloud obtained from drone photogrammetry. This registration can be done using automatically extracted corresponding features in Agisoft Metashape ${ }^{\circledR}$, or by manually measuring tie points.

As final outputs, three point clouds were produced, one per each observation epoch in 2016, 2017 and 2018, respectively (Fugazza et al., 2018; Scaioni et. 2019). The merged point clouds from 2016 data sets was made up of 4.38 million points after resampling (see Fig. 2). Figure 3 represents the point cloud obtained from 2017 data sets (85.30 million points before resampling), and Figure 4 the one from 2018 data sets (146.07 million points before resampling). All point clouds are colored using RGB information from images.

\subsection{Comparison of point clouds}

All point clouds were resampled in CloudCompare (2019) environment at $10 \mathrm{~cm}$ minimum linear distance between two closest points. Since the merged point clouds accounted for abundant points in those overlapping regions reconstructed from both "terrestrial” and "drone" photogrammetric blocks, subsampling helped get rid of highly redundant data and make them easier to handle. In additional, this step allowed to standardize all point clouds to a common spatial resolution.

Different types of comparison were done in CloudCompare and QGIS (2019), as described in the following paragraphs. Indeed, a standard technique to compare a pair of point clouds is to resample it to the nodes of a regular grid to obtain a digital elevation model (DEM), which may be referred to as a 2.5D data set (Gu et al., 2014).

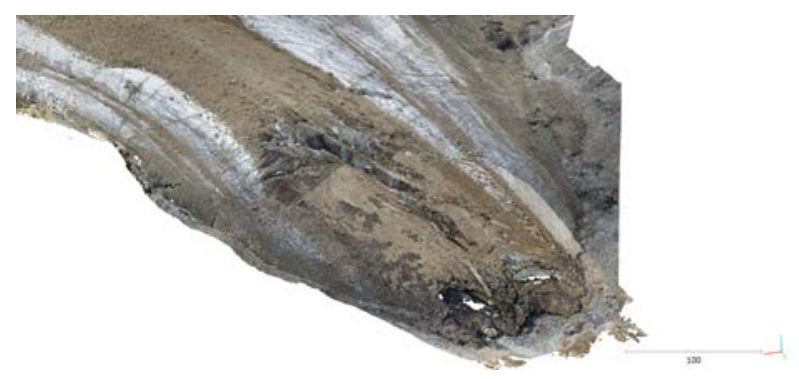

Figure 2. Final point cloud obtained from 2016 campaign at Forni Glacier (4.38 million points after resampling).

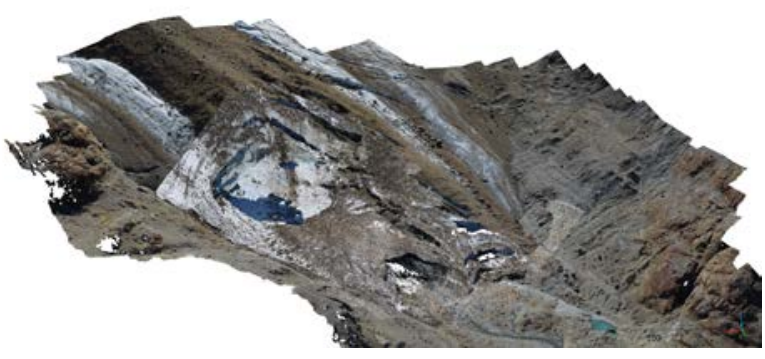

Figure 3. Final point cloud obtained from 2017 campaign at Forni Glacier (85.30 million points).

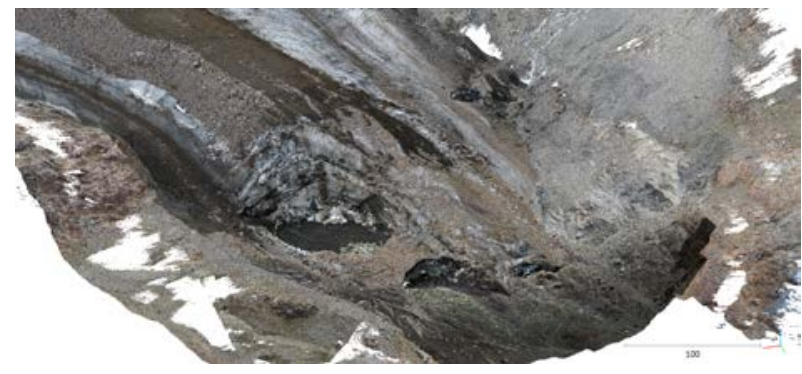

Figure 4. Final point cloud obtained from 2018 campaign at Forni Glacier (146.07 million points).

On the other hand, when DEMs are compared, they only allow to highlight those elevation changes that happen in the direction perpendicular to the reference plane, which in general is horizontal. Consequently, this approach (see Par. 3.2.1) may provide sound results when the entire glacier surfaces are compared, as demonstrated in Fugazza et al. (2018). Of course, when the investigated region features a much more complex surface morphology, such as the case of glacier terminus, the analysis of DEMs may not provide significant outcomes. At Paragraph 3.2.2 an alternative method (M3C2) to compare two point clouds that does not rely on DEMs but that is directly based on raw point clouds is applied.

3.2.1 Comparison of DEMs. CloudCompare allows the creation of a DEM from a point cloud on the basis of the 'Rasterize' tool. Once created according to a specific grid resolution, the obtained DEM can be exported using GEOTIFF format, which can be used later in GIS environment for further analysis (pairwise comparison, visualization, contour line generation, etc.).

After exporting the DEMs for all three years, they have been imported in QGIS, where simple raster calculation has 
been performed to obtain the absolute elevation difference between pairs of resampled clouds (see Figures 5 and 6).
From the analysis of DEMs it is easy to derive volumetric changes. The 2016-2017 volume comparison resulted in losing a volume of $960.6 \cdot 10^{3} \mathrm{~m}^{3}$ of ice, while the comparison between 2017-2018 DEMs showed a loss of $391.3 \cdot 10^{3} \mathrm{~m}^{3}$.

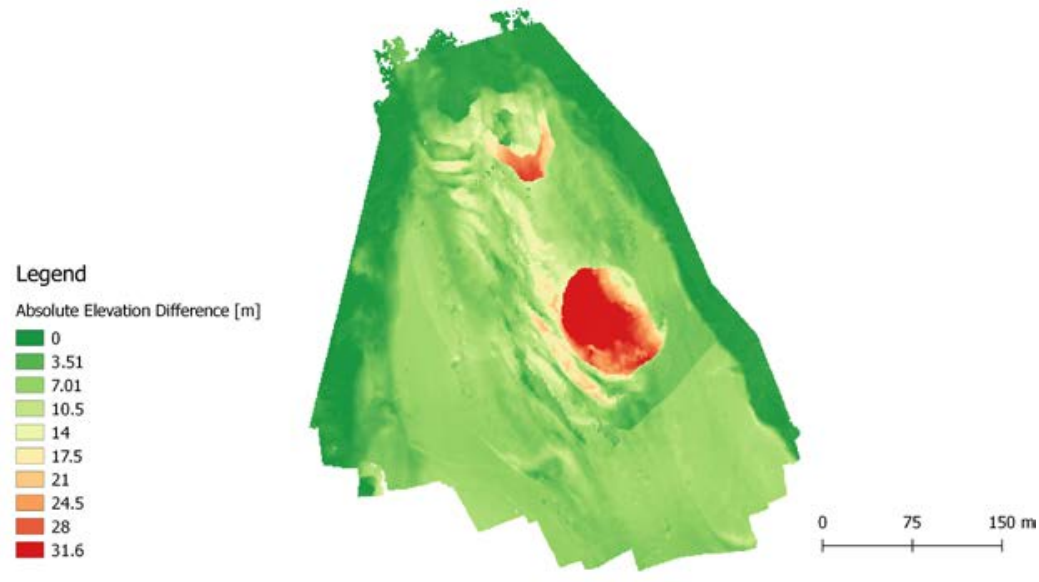

Figure 5. Absolute elevation difference between 2016 and 2017 DEMs (corresponding volume change: 960.6·10³ $\mathrm{m}^{3}$ ).

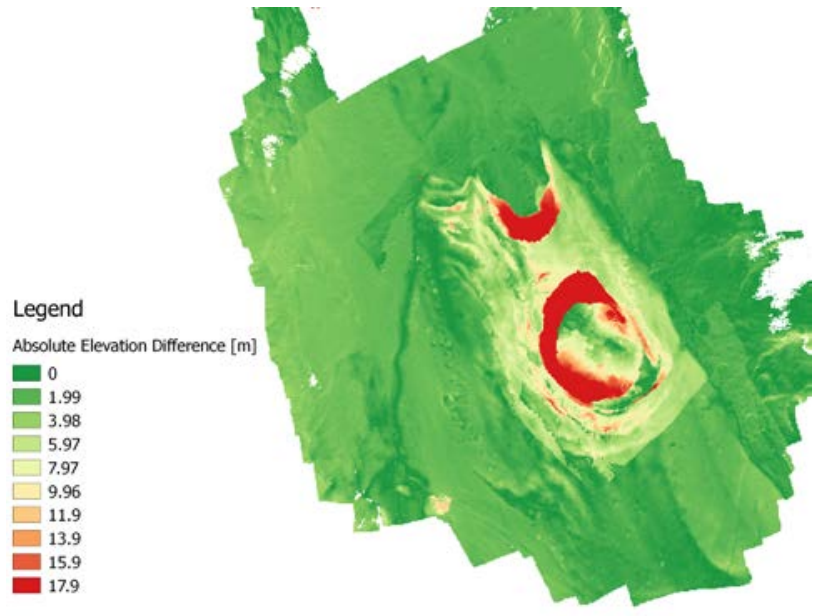

Figure 6. Absolute elevation difference between 2017 and 2018 DEMs (corresponding volume change: $391.3 \cdot 10^{3} \mathrm{~m}^{3}$ ).

3.2.2 M3C2. M3C2 tool developed by Lague et al. (2013) provides direct cloud-to-cloud comparison considering the local normal directions and the local roughness. This method may also receive as input the registration error and the point cloud precision (see, e.g., James et al., 2017) without the need of meshing or grid resampling. M3C2 is able to distinguish surface displacements in multiple directions, and then is more suitable to analyse objects with a complex 3D shape as the glacier terminus under investigation.

Two comparisons have been done between point clouds from 2016 and 2017, and between point clouds from 2017 and 2018. The results from M3C2 comparison are reported in Figures 7 and 8 . The first notable change is the major hole that has collapsed between campaigns in 2016 and 2017, resulting in depression deeper more than $50 \mathrm{~m}$ from the glacier surface. The surroundings areas of the glacier are depicted in orange, colour that means no difference is present. On the other hand, the thinning of the glacier tongue is prominent in the yellow to green areas which represent elevation loss from 4 to $20 \mathrm{~m}$. Looking at Figures 7 and 8, one can notice that the difference depicted in the period 20172018 is not so easily notable except the thinning of the ice 'bridge' next to hole. On the other hand, Figure 6 clearly represents again the tongue thinning in elevation, corresponding to a loss less than $10 \mathrm{~m}$, and the hole expansion with more than $20 \mathrm{~m}$. 


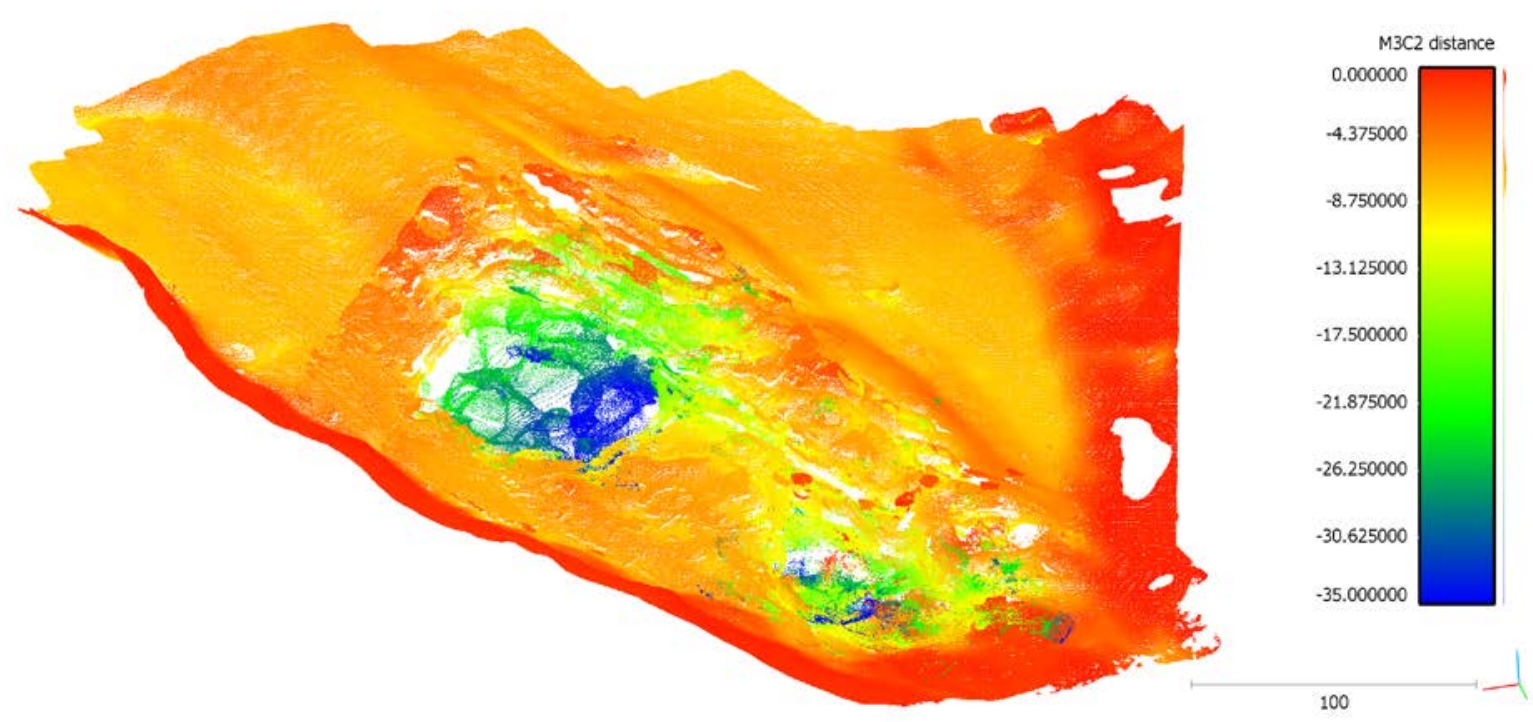

Figure 7. M3C2 comparison between '2016' and '2017' point clouds (differences in metres).
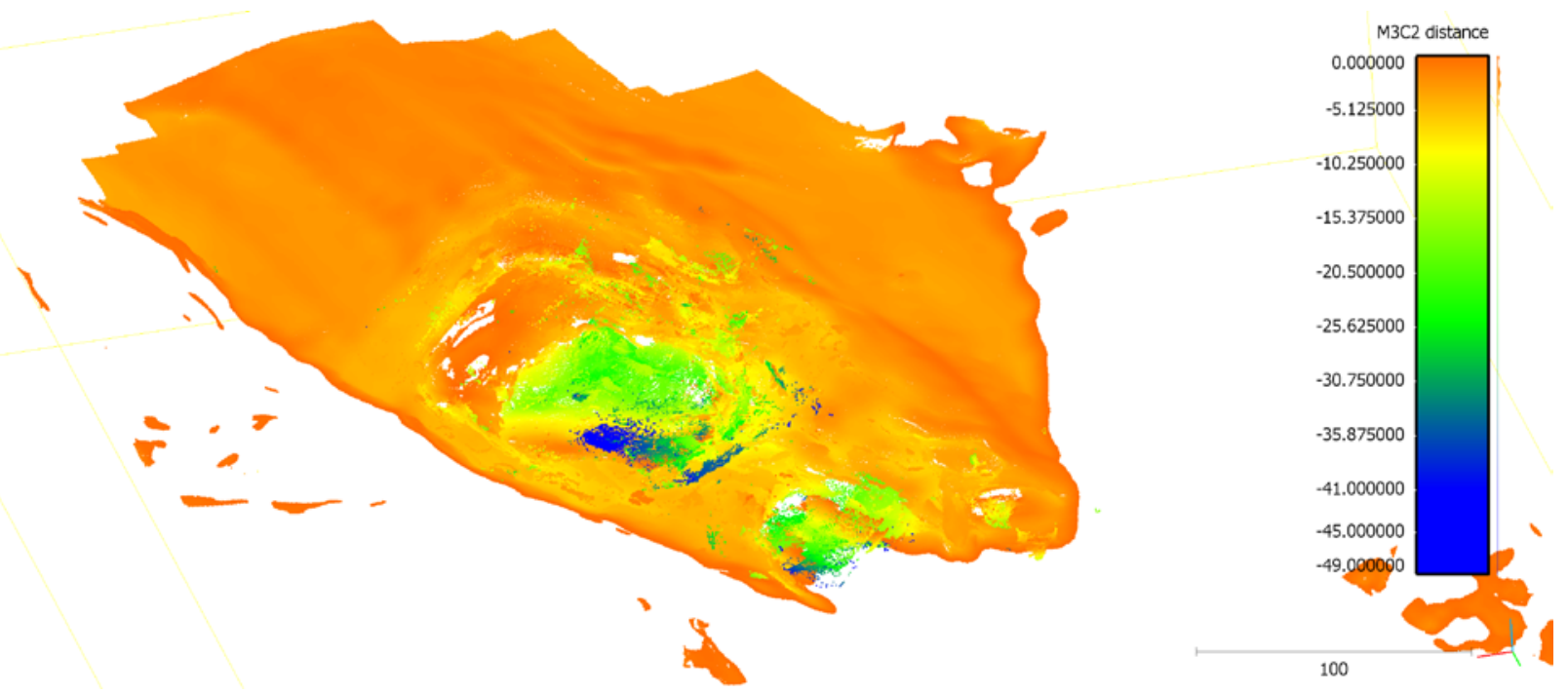

Figure 8. M3C2 comparison between '2017’ and '2018’ point clouds (differences in metres).

\subsection{Sentinel 2A/2B medium resolution satellite images}

Figure 9 shows some patches from a series of multispectral medium-resolution satellite images over the Forni Glacier from constellation Sentinel 2 (ESA, 2019). Data spans over a period from 2016 and 2018, which is contemporary to the acquisition of near-sensing data sets described in Subsection 3.1. Images only refer to summer because of the snow and cloud cover that prevented the analysis of winter data.

Despite of the large GSD (10 m), Sentinel 2 images have been used to provide an overview of the evolution of the retreat in the period that is considered here. In addition, they allow to understand the formation and development of the local collapses close to the terminus of the glacier tongue.

\section{CONCLUSIONS}

In this paper different data sets collected using different remote sensing platforms to study an Alpine Glacier (Forni
Glacier, Italian Alps) have been presented. The aim was to describe an integrated approach to analyze the morphological changes in the area of the glacier terminus, in order to highlight global and local changes.

In particular, a procedure for the photogrammetric reconstruction based on "terrestrial" and "drone" photos is presented. First data are processed using Structure-fromMotion. Secondly those point clouds obtained after dense surface matching are compared. In the future, archive photos will be integrated in the analysis to reconstruct changes in the past years.

On the other side, a time-series of medium resolution satellite imagery (Sentinel 2) has been considered to analyze the general changes on the glacier surface. This approach can be integrated to the in-situ surveying campaign to study the long-term evolution of the glacier dynamics. In future, the analysis of ice-flow velocity will be done, also include the use of radar data collected by Sentinel constellation. 

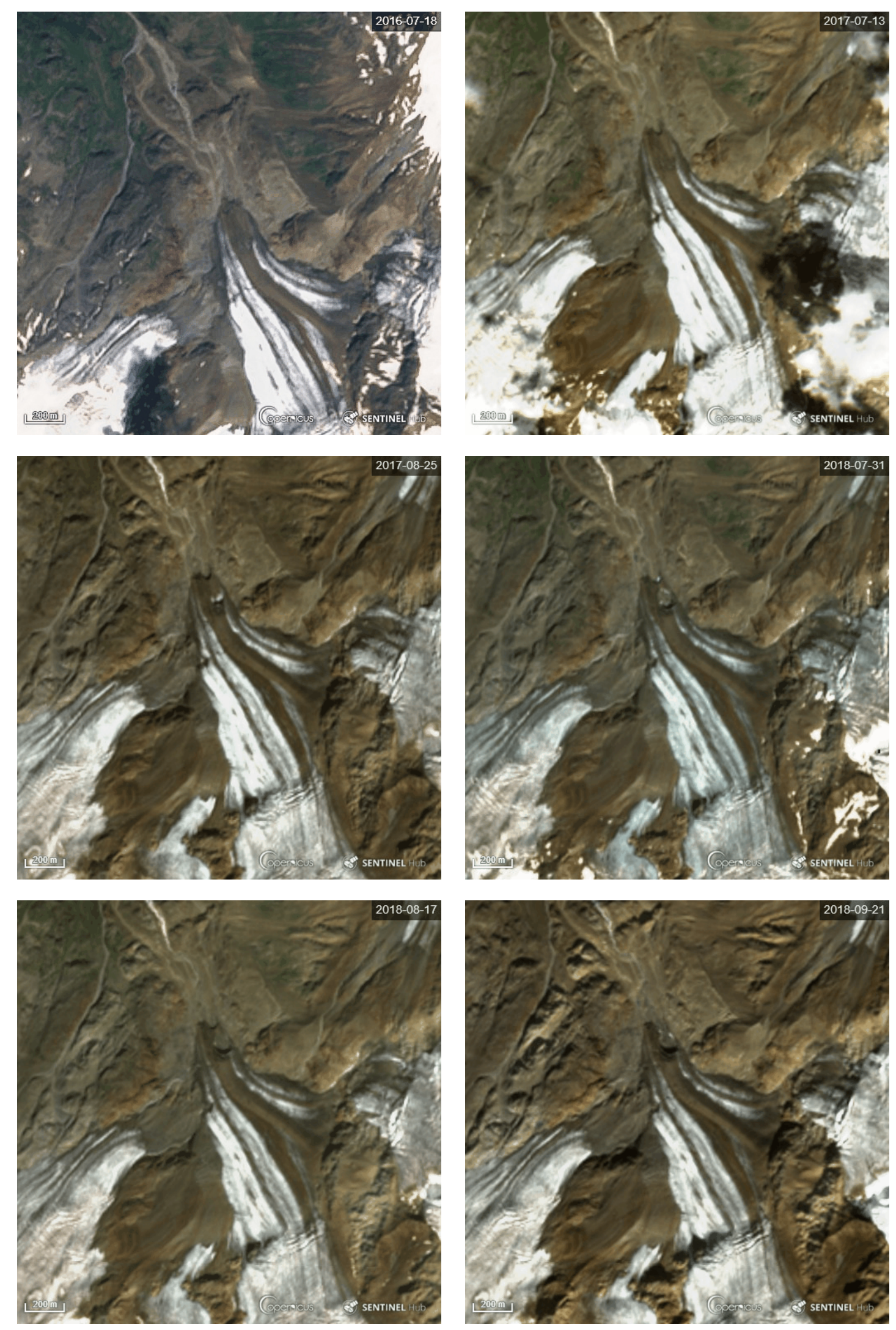

Figure 9. Series of Sentinel 2 images over the area of Forni Glacier from 2016-2018. 


\section{Acknowledgements}

This study was funded by DARA (Department for regional affairs and autonomies) of the Presidency of the Council of the Italian Government). The authors acknowledge the Central Scientific Committee of CAI (Italian Alpine Club) and Levissima San Pellegrino S.P.A. for funding the UAV quadcopter, and Stelvio Park Authority for the logistic support and for permitting the UAV surveys. Acknowledgements go to those colleagues, students and friends who helped with different stages of field operations. In particular Manuel Corti and Julién Crippa. The authors would like to acknowledge the ESA Copernicus program for delivering Sentinel images under open-data policy, Agisoft company (Russia) for granting one-month free evaluation license of software Metashape ${ }^{\circledR}$, and to CloudCompare and QGIS developers.

\section{REFERENCES}

Atkinson, K.B. (1996). Close Range Photogrammetry and Machine Vision. Whittles Publishing, Caithness, Scotland, 371 pages.

Azzoni, R.S., Senese, A., Zerboni, A., Maugeri, M., Smiraglia, C., Diolaiuti, G.A. (2016). Estimating Ice Albedo from Fine Debris Cover Quantified by a Semi-Automatic Method: The Case Study of Forni Glacier, Italian Alps. Cryosphere, 10(2): 665-679.

Azzoni, R.S., Fugazza, D., Zennaro, M., Zucali, M., D’Agata, C., Maragno, D., Cernuschi, M., Smiraglia, C., Diolaiuti, G.A. (2017). Recent structural evolution of Forni Glacier tongue (Ortles-Cevedale Group, Central Italian Alps). J. Maps, 13(2): 870-878, doi: 10.1080/17445647.2017.1394227.

Azzoni, R.S., Fugazza, D., Zerboni, A., Senese, A., D’Agata, C., Maragno, D., Cazzaniga, A., Cernuschi, M., Diolaiuti, G.A. (2018). Evaluating High-Resolution Remote Sensing Data for Reconstructing the Recent Evolution of Supra Glacial Debris:

A Study in the Central Alps (Stelvio Park, Italy). Progr. Phys. Geog. Earth Env., 42(1): 3-23.

Barazzetti, L., Remondino, F., Scaioni, M., Brumana, R. (2010). Fully Automatic UAV Image-Based Sensor Orientation. Int. Arch. Photogramm. Remote Sens. Spatial Inf. Sci., Vol. 38, Part 1, 6 pp.

CloudCompare (2019). Cloudcompare version 2.9.1 GPL software. Available online at: www.cloudcompare.org/ (last accessed on $2^{\text {nd }}$ April 2019).

Cogliati, M., Tonelli, E., Battaglia, D., and Scaioni, M., 2017. Extraction of DEMs and Orthoimages from Archive Aerial Imagery to Support Project Planning in Civil Engineering. ISPRS Ann. Photogramm. Remote Sens. Spatial Inf. Sci., Vol. IV, Part 5/W1, pp. 9-16.

Diolaiuti, G.A., Smiraglia, C., 2010. Changing glaciers in a changing climate: How vanishing geomorphosites have been driving deep changes in mountain landscapes and environments. Geomorphologie: relief, processus, environnement, 16:131-152.

Eltner, A., Kaiser, A., Castillo, C., Rock, G., Neugirg, F., Abellán, A., 2015. Image-based surface reconstruction in geomorphometry - merits, limits and developments. Earth Surface Dynamics, 4: 359-389.

ESA (2019). Sentinel online. Available online at: https://sentinel.esa.int/web/sentinel/missions/sentinel-2 (last accessed on $2^{\text {nd }}$ April 2019).

Feurer, D., Vinatier, F., 2018. Joining multi-epoch archival aerial images in a single SfM block allows $3-\mathrm{D}$ change detection with almost exclusively image information. ISPRS J. Photogramm. Remote Sens., 146: 495-506.

Forlani, G., Roncella, R., Diotri, F., 2015. Production of high-resolution digital terrain models in mountain regions to support risk assessment. Geomat., Nat. Haz. Risk, 6(5-7): 379-397.

Fugazza, D., Senese, A., Azzoni, R.S., Maugeri, M., Diolaiuti, G.A. (2016). Spatial Distribution of Surface Albedo at the Forni Glacier (Stelvio National Park, Central Italian Alps). Cold Reg. Sci. Tech., 125: 128-137.

Fugazza, D., Scaioni, M., Corti, M., D’Agata, C., Azzoni, R.S., Cernuschi, M., Smiraglia, C., Diolaiuti, G.A., 2018. Combination of UAV and terrestrial photogrammetry to assess rapid glacier evolution and map glacier hazards. Nat. Hazards Earth Syst. Sci., Vol. 18, pp. 1055-1071, DOI: 10.5194/nhess-18-1055-2018.

Fugazza, D. (2019). All eyes on glaciers: remote sensing of the cryosphere. PhD Thesis at University of Milan, Dept. of Earth Sciences “A. Desio.”

Giordan, D., Hayakawa, Y., Nex, F., Remondino, F., Tarolli, P. (2018). Review article: the use of remotely piloted aicrafts systems (RPASs) for natural hazards monitoring and assessment. Nat. Hazards Earth Syst. Sci., 18: 1079-1096.

Granshaw, S.I., 2018a. RPV, UAV, UAS, RPAS ... or just drone? Photogramm. Rec., 32(162): 160-170.

Granshaw, S.I., 2018b. Structure from Motion: Origins and Originality. Photogramm. Rec., 33(161): 6-10.

Gu, Z., Feng, T., Scaioni, M., Wu, H., Liu, J., Tong, X., Li, R. (2014). Experimental Results of Elevation Change Analysis in the Antarctic Ice Sheet Using DEMs from ERS and ICESat Data. Ann. Glaciol., 55(66):198-204, DOI: 10.3189/ 2014AoG66A124.

James, M.R., Robson, S., Smith, M.W. (2017). 3-D uncertainty-based topographic change detection with structure-from-motion photogrammetry: precision maps for ground control and directly georeferenced surveys. Earth Surf. Proc. Landforms, 42(12): 1769-1788.

Lague, D., Brodu, N., Leroux, J. (2013). Accurate 3D comparison of complex topography with terrestrial laser scanner: Application to the Rangitikei canyon (NZ). ISPRS J. Photogramm. Remote Sens., 82:10-26.

Li, R., Ye, W., Qiao, G., Tong, X., Liu, S., Kong, F., and X. Ma, 2017. A New Analytical Method for Estimating Antarctic Ice Flow in the 1960s from Historical Optical Satellite Imagery. IEEE Trans. Geosci. Remote Sens., 55(5): 2771-2785.

Luhmann, T., Robson, S., Kyle, S., Boehm, J., 2014. Close Range Photogrammetry: 3D Imaging Techniques - 2nd Edition. Walter De Gruyter Inc., Germany, 684 pages.

Mosbrucker, A.R., Major. J.J., Spicer, K.R., Pitlick, J. (2017). Camera system considerations for geomorphic 
applications of SfM photogrammetry. Earth Surf. Proc. Land., 42:969-986.

O’Connor, J., Smith, M.J., James, M.R. (2017). Cameras and settings for aerial surveys in the geosciences: Optimising image data. Prog. Phys. Geog., 41:325-344.

Paul, F., Winsvold, S.H., Kääb, A., Nagler, T., Schwaizer, G. (2016). Glacier Remote Sensing Using Sentinel-2. Part II: Mapping Glacier Extents and Surface Facies, and Comparison to Landsat 8. Remote Sensing, 8(7): 575-590.

Pepe, M., Fregonese, L., Scaioni, M. (2018). Planning airborne photogrammetry and remote-sensing missions with modern platforms and sensors. Eur. J. Remote Sens., 51:412435, DOI:10.1080/22797254.2018.1444945.

QGIS (2019). QGIS Geographic Information System. Open Source Geospatial Foundation Project.

Rutzinger, M., Bremer, M., Höfle, B., Hämmerle, M., Lindenbergh, R., Oude Elberink, S., Pirotti, F., Scaioni, M., Wujanz, D., Zieher, T. (2018). Training in Innovative Technologies for Close-Range Sensing in Alpine Terrain. ISPRS Ann. Photogramm. Remote Sens. Spatial Inf. Sci., Vol. IV, Part 2, pp. 239-246, DOI: 10.5194/isprs-annals-IV-2-2392018.

Rutzinger, M., Höfle, B., Lindenbergh, R., Oude Elberink, S., Pirotti, F., Sailer, R., Scaioni, M., Stötter, J., Wujanz, D. (2016). Close-Range Sensing Techniques in Alpine Terrain. ISPRS Ann. Photogramm. Remote Sens. Spatial Inf. Sci., Vol. III, Part 6, pp. 15-22, DOI: 10.5194/isprs-annals-III-6-152016.

Scaioni, M., Corti, M., Diolaiuti, G., Fugazza, D., Cernuschi, M. (2017). Local and general monitoring of Forni glacier (Italian alps) using multi-platform structure-from-motion photogrammetry. Int. Arch. Photogramm. Remote Sens. Spatial Inf. Sci. Vol. 42, Part 2/W7:1547-1554, DOI:10.5194/isprs-archives-XLII-2-W7-1547-2017.

Scaioni, M., Barazzetti, L., Yordanov, V., Azzoni, R.S., Fugazza, D., Cernuschi, M., Diolaiuti, G.A., 2019. "Structure-From-Motion Photogrammetry to Support the Assessment of Collapse Risk in Alpine Glaciers.” In: O. Altan, M. Chandra, F. Sunar, T. Tanzi T. (Ed.'s), Intelligent Systems for Crisis Management. Gi4DM 2018, Lecture Notes in Geoinformation and Cartography. Springer, Cham (Switzerland), pp. 239-263.

Scaioni, M., Crippa, J., Corti, M., Barazzetti, L., Fugazza, D., Azzoni, R., Cernuschi, M., Diolaiuti, G.A. (2018). Technical Aspects Related to the Application of SfM Photogrammetry in High Mountain. Int. Arch. Photogramm. Remote Sens. Spatial Inf. Sci., Vol. XLII, Part 2, pp. 1029-1036, DOI: 10.5194/isprs-archives-XLII-2-1029-2018.

Shan, J., Toth, C.K. (2009). Topographic Laser Scanning and Ranging. Principles and Processing. Taylor and Francis Group, Boca Raton, FL-USA.

Vosselman, G., Maas, H.-G (2010). Airborne and Terrestrial Laser Scanning. Taylor and Francis Group, Boca Raton, FLUSA.

Walstra, J., Dixon, N., Chandler, J.H., 2007. Historical aerial photographs for landslide assessment: two case histories. Quart. J. Eng. Geol. Hydrogeol., 40. 315-332. 